TRANSACTIONS OF THE

AMERICAN MATHEMATICAL SOCIETY

Volume 195, 1974

\title{
SEMIGROUPS OVER TREES
}

\author{
BY
}

M. W. MISLOVE

ABSTRACT. A semigroup over a tree is a compact semigroup $S$ such that $H$ is a congruence on $S$ and $S / \mathcal{H}$ is an abelian tree with idempotent endpoints. Each such semigroup is characterized as being constructible from cylindrical subsemigroups of $S$ and the tree $S / H$ in a manner similar to the construction of the hormos. Indeed, the hormos is shown to be a particular example of the construction given herein when $S / \mathfrak{H}$ is an $I$-semigroup. Several results about semigroups whose underlying space is a tree are also established as lemmata for the main results.

Introduction. Recall that a tree is a continuum in which any two points can be separated by a third point. In [3], Hofmann and Mostert prove the following:

Theorem. Let $S$ be a compact semigroup. $\mathcal{H}$ is a congruence on $S$ and $S / \mathcal{H}$ is an l-semigroup if and only if $S=\operatorname{Horm}\left(X, S_{x}, m_{x y}\right)$ for some chainable collec. tion $\left(X, S_{x}, m_{x y}\right)$.

This theorem completely describes the semigroup $S$ in terms of $S / \mathcal{H}$ and cylindrical subsemigroups of $S$. Our purpose here is to generalize this result by obtaining a similar characterization of those compact semigroups $S$ with $S / \mathcal{H}$ an abelian tree with idempotent endpoints, thus giving a partial solution to Problem 43 of $[1$, p. 99] and also to Problem P5 of [3, p. 160].

If $\left\{x_{a}\right\}_{a \in D}$ is a net in a space $X$ and $x \in X,\left\{x_{a}\right\}_{a \in D} \stackrel{f}{\rightarrow} x,\left(\left\{x_{a}\right\}_{a \in D} \stackrel{e}{\rightarrow} x\right)$ will denote the fact that $\left\{a \in D: x_{a} \in U\right\}$ is cofinal (residual) in $D$ for each open set $U$ containing $x$. Otherwise, the notation and terminology will be that of [3]. This work forms part of the author's doctoral dissertation, and he wishes to express his deep gratitude to Professor J. H. Carruth for his many helpful suggestions and his advice, and for his patient listening during its preparation.

The following will be referred to as Koch's theorem throughout this work.

Theorem (Koch [6]). Let $S$ be a compact connected semigroup with identity 1 and minimal ideal $M(S) \neq S$. If each subgroup of $S$ is totally disconnected, then there is a standard thread $I$ in $S$ from 1 to $M(S)$.

Presented to the Society, November 12, 1969; received by the editors October 20, 1970 and, in revised form, November 27, 1973.

AMS (MOS) subject classifications (1970). Primary 22A15.

Key words and phrases. Compact semigroup, tree, semigroup over a tree, generalized collection, hormos, l-semigroup. 
Semigroups on trees. The structure of trees and of semigroups whose underlying space is a tree has been studied in [7], [12], [13], and [14]. We now list some properties established in these works, and we shall use these properties throughout this work without specific reference.

If $X$ is a continuum, $X$ is a tree if and only if $X$ is hereditarily unicoherent and locally connected [13]. If $X$ is a tree and $p \in X$, define $\leqq_{p}$ on $X$ by $x \leqq_{p} y$ if and only if $x=y, x=p$, or $x$ separates $y$ and $p$. If $x \in X$, let $M(x)=\left\{y \in X: x \leqq_{p} y\right\}$ and $L(x)=$ $\left\{y \in X: y \leqq_{p} x\right\}$. Then, $\leqq_{p}$ is a closed partial order on $X, M(x)$ and $L(x)$ are closed subsets of $X$, and $M(x)-\{x\}$ is anopen subset of $X$ for each $x \in X$ [14]. If $A$ and $B$ are connected subsets of $X$, then $A \cap B$ is connected. If $a, b \in X$, there is a unique arc in $X$ from $a$ to $b$, denoted $[a, b]$, and $[a, b]=\{a, b\} \cup$ $\{x \in X: x$ separates $a$ and $b\}$. If $a \in X$ and $\left\{x_{a}\right\}_{a \in D} \subset X$ with $\left\{x_{a}\right\}_{a \in D} \stackrel{e}{\rightarrow} x \in X$, then $\left\{\left[a, x_{a}\right]\right\}_{a \in D} \stackrel{e}{\rightarrow}[a, x]$, where convergence in the latter case is in the $\lim$ sup-lim inf sense [7]. From this it is easily shown that if $\left\{y_{a}\right\}_{a \in D} \subset \underset{e}{ }$, $\left\{y_{a}\right\}_{a \in D} \stackrel{e}{\rightarrow} y \in X$, and $y_{a} \in\left[a, x_{a}\right]$ for each $a \in D$, then $\left\{\left[y_{\infty}, x_{a}\right]\right\}_{a \in D} \stackrel{e}{\rightarrow}[y, x]$. Finally, each subcontinuum of a tree is itself a tree [12].

Definition 1.1. Let $T$ be a tree. $x \in T$ is an endpoint of $T$ if $x$ separates no $\operatorname{arc}$ in $T$.

Lemma 1.2. Let $T$ be a tree and let $x \in T$. If $x$ is not a cutpoint of $T$, then $x$ is an endpoint of $T$.

Proof. If $A$ is an arc with endpoints $a$ and $b$, then $A=[a, b]$ since $T$ is uniquely arcwise connected. If $x$ separates $A$, then $x \in(a, b)$, whence $x$ separates $a$ and $b$. But, in that case, $x$ sepatates $T$, and so $x$ is a cutpoint of $T$. The result follows by contraposition.

Our concern will be with semigroups on trees with idempotent endpoints in which the idempotents commute. The following results show we can assume that the trees with which we work are abelian.

Lemma 1.3 (Hunter [4]). Suppose $T$ is a semigroup with zero on a bereditarily unicoberent arcwise connected continuum. If the endpoints of $T$ commute, one with another, then $T$ is abelian.

Lemma 1.4. Suppose $T$ is a semigroup on a tree with idempotent endpoints in which the idempotents commute. Then the maximal subgroups of $T$ are totally disconnected, and bence $T$ has a zero.

Proof. If $e \in E(T)$, then $H_{0}(e)$, the identity component of $H(e)$, is a subcontinuum of $T$, and so it is a tree. Thus, $H_{0}(e)=\{e\}$ by homogeneity, whence $H(e)$ is totally disconnected.

If, now, $e \in E(T) \cap M(T)$, then $H(e)=e T e$ is connected and totally disconnected, and so $H(e)=\{e\}$. Thus, $M(T) \subset E(T)$, and we have $M(T)$ is a singleton, since $E(T)$ is abelian. 
Lemma 1.5. If $T$ is an abelian semigroup on a tree with idempotent endpoints, then $[0, e]$ is a standard thread and $H(e)=\{e\}$ for each $e \in E(T)$. Consequently, if $s \in[0, t]$, then $s \leqq_{\mathcal{H}} t$.

Proof. According to Lemma 1.4, each maximal subgroup of $T$ is totally disconnected, and so, if $e \in E(T)$, then Koch's theorem implies the existence of a standard thread $I$ running from $e$ to 0 . But, since $[0, e]$ is the unique arc in $T$ from $e$ to $0, I=[0, e]$.

Now, since $T$ has idempotent endpoints, $T=\bigcup\{[0, f]: f \in E(T)\}$, and so $e T e=\bigcup\{[0, e f]: f \in E(T)\}$. Thus, as $e T e$ is a subcontinuum of $T, e T e$ is a tree with idempotent endpoints, and, by [7], no point of $H(e)$ is a cutpoint of $T$. Hence, $H(e)=\{e\}$ by Lemma 1.2.

Finally, if $s \in[0, t]$, then $s, t \in[0, e]$ for some $e \in E(T)$ since $T$ has idempotent endpoints. Then, $s \in t[0, e] \cap[0, e] t \subset t T \cap T t$, whence $s \leqq_{\mathcal{H}} t$.

Let $T$ be an abelian semigroup on a tree with idempotent endpoints, and let 0 be the zero of $T$. The relation defined on $T$ by $x \leqq y$ if and only $x \in[0, y]$ will be called the cutpoint order on $T$, and if $x \in T, M(x)$ and $L(x)$ will denote the upper and lower sets at $x$, respectively, with respect to this order only. Since $\leqq$ is a closed partial order on $T, T$ is locally convex with respect to $\leqq$ [10, Proposition 3 and Corollary 4]. If $X=E(T)$, we define $X^{\prime}=\{x \in X: x$ is isolated in $[0, x] \cap X\}$, and if $x \in X^{\prime}$, we let $x^{\prime}=\sup ([0, x) \cap X)$. We shall also use this notation consistently throughout this work. We now establish some convergence properties in $T$.

Proposition 1.6. Let $T$ be an abelian semigroup on a tree with idempotent endpoints, let $X=E(T)$, and let $\left\{x_{a}\right\}_{a \in D} \subset X$ with $\left\{x_{a}\right\}_{a \in D} \stackrel{e}{\rightarrow} x \in X$.

(a) If $x_{a} \in X^{\prime}$ for each $a \in D, x \in X^{\prime}$, and $\left\{x_{a}^{\prime}\right\}_{a \in D} \stackrel{e}{\rightarrow} x^{\prime}$, then there is $\beta \in D$ with $x_{a}^{\prime}=x^{\prime}$ for $\alpha \geqq \beta$.

(b) If $x \in X^{\prime}$ and $x_{a} x=x_{a}$ for each $\alpha \in D$, then there is $\beta \in D$ with $x_{a} \in X^{\prime}$ and $x_{a}^{\prime} \in X^{\prime}$ for each $a \geqq \beta$.

Proof. For part (a), if $t \in\left(x^{\prime}, x\right)$, then $x \in M(t)-\{t\}$ is open in $T$. Thus, there is $\beta_{1} \in D$ with $x_{a} \in M(t)-\{t\}$ for $\alpha \geqq \beta_{1}$, and so $[0, i] \subset\left[0, x_{\alpha}\right]$ for $\alpha \geqq$ $\beta_{1}$. Now, $M(t)$. is closed, and, as $x^{\prime} \in T-M(t)$, there is $\beta_{2} \in D$ with $x_{a}^{\prime} \epsilon$ $T-M(t)$ for $\alpha \geqq \beta_{2}$, whence $x_{a}^{\prime} \in[0, t]$ for $\alpha \geqq \beta_{1}, \beta_{2}$. Thus, if $\beta \in D$ with $\beta \geqq \beta_{1}, \beta_{2}$, then $x_{a}^{\prime}=x^{\prime}$ for $\alpha \geqq \beta$ since $\left[x^{\prime}, t\right] \cap X=\left\{x^{\prime}\right\}$.

For part (b), suppose $x_{\alpha} x=x_{a}$ for each $a \in D$ and $x \in X^{\prime}$. Then, $x \in M\left(x^{\prime}\right)$ $-\left\{x^{\prime}\right\}$ and $M\left(x^{\prime}\right)-\left\{x^{\prime}\right\}$ is an open subset of $T$, and so there is $\beta \in D$ with $x_{a}$ $\in M\left(x^{\prime}\right)-\left\{x^{\prime}\right\}$ for $\alpha \geqq \beta$, whence $x^{\prime} \in\left[0, x_{\alpha}\right]$ for $\alpha \geqq \beta$. Now, for $\alpha \geqq \beta$, $x_{a}\left[x^{\prime}, x\right]=\left[x_{a^{\prime}} x^{\prime}, x_{\alpha}\right]$; and, as $x^{\prime} \in\left[0, x_{\alpha}\right), x_{\alpha} x^{\prime}=x^{\prime}$ by Lemma 1.5. Moreover, as $T$ is abelian and $x_{a}$ is idempotent, translation by $x_{\alpha}$ is a homomorphism, 
and hence $\left(x^{\prime}, x_{a}\right) \cap X=\square$. Thus, for $a \geqq \beta, x_{a} \in X^{\prime}$ and $x^{\prime}=x_{a}^{\prime}$, concluding the proof.

Lemma 1.7. Let $T$ be an abelian semigroup on a tree and let $X=E(T)$. If $x \in X^{\prime}$ and $x$ is not isolated in $x X$, then

$$
D=\{y \in X: x y=y \text { and } y \text { is isolated in } y X\}
$$

is a directed set under $y \leqq z$ if and only if $y z=y$, and $\{y\}_{y \in D} \stackrel{e}{\rightarrow} x$.

Proof. If $y_{1}, y_{2} \in D$, then, for $i=1,2, y_{i} x=y_{i}$ and $y_{i}$ isolated in $y_{i} X$ imply there is an open set $U$ containing $x$ with $y_{i} U \cap X=\left\{y_{i}\right\}$ for each $i$. But $x$ is not isolated in $x X$ and $x \in X^{\prime}$, whence a simple application of Koch's theorem yields the existence of $y \in U-\{x\}$ with $y x=y$ and $y$ isolated in $y X$. Clearly $y \in D$ and $y_{1}, y_{2} \leqq y$.

To show $\{y\}_{y \in D} \stackrel{e}{\rightarrow} x$, it suffices to show this convergence in $X$. But, since $\leqq$ is a closed partial order on $X, X$ is locally convex with respect to $\leqq$. Now, if $x \in U$ and $U$ is open and convex, a simple application of Koch's theorem yields $D \cap U \neq \square$, and if $y \in D \cap U$ and $z \in D$ with $y \leqq z$, then $y \leqq z \leqq x$, and so $z \in U$ by convexity. This proves the result.

Semigroups over trees. We now turn our attention to the first of our main results. The following definition is very similar to that of a chainable collection [3, p. 139].

Definition 2.1. ( $\left.T, X, S_{x}, m_{x y}, \eta_{x}\right)$ is a generalized collection if:

(a) $T$ is an abelian semigroup on a tree with idempotent endpoints and $X=E(T)$.

(b) For each $x \in X, S_{x}$ is a cylindrical semigroup with identity $1_{x}$ and minimal ideal $M_{x}$ satisfying:

(i) If $x \notin X^{\prime}$, then $S_{x}=H_{x}=M_{x}$ is a group, $H_{x}$ being the group of units of $S_{x^{*}}$

(ii) If $x \in X^{\prime}, \eta_{x}: S_{x} \rightarrow\left[x^{\prime}, x\right]$ is a surmorphism, and there is an isomorphism $\psi_{x}: S_{x} / \mathcal{H}_{x} \rightarrow\left[x^{\prime}, x\right]$ so that $\psi_{x} \nu_{x}=\eta_{x}$, where $\nu_{x}: S_{x} \rightarrow S_{x} / \mathcal{H}_{x}$ is the natural map.

(iii) If $x \neq y$, then $S_{x} \cap S_{y}=\square$.

(c) If $x, y \in X$ with $x y=x$, then $m_{x y}: S_{y} \rightarrow S_{x}$ is a homomorphism with:

(i) $m_{x x}$ is the identity.

(ii) If $x \in[0, y)$, then $m_{x y}\left(S_{y}\right) \subset H_{x}$.

(iii) If $x y=x$ and $y z=y$, then $m_{x y} \circ m_{y z}=m_{x z^{\circ}}$

(d) (i) If $x \in X^{\prime}$, then $m_{x^{\prime} x} \mid M_{x}$ is an injection.

(ii) If $x, y \in X^{\prime}, x y=x$, and $x^{\prime}=y^{\prime}$, then $m_{x y} \mid \eta_{y}^{-1}\left[y^{\prime}, t\right]$ is an injection into $\eta_{x}^{-1}\left[x^{\prime}, t\right]$, where $t=\sup \left(\left[x^{\prime}, x\right] \cap\left[y^{\prime}, y\right]\right)$.

(iii) Suppose $\left\{x_{a}\right\}_{a \in D} \subset X$ with $\left\{x_{a}\right\}_{a \in D} \stackrel{e}{\rightarrow} x$ so that $x_{a} x=x_{a}$ for each $a \in D$ 
and $x_{a} x_{\beta}=x_{a}$ if $a \leqq \beta \in D$. Then, $\phi_{x}: S_{x} \rightarrow \Pi\left\{S_{x_{a}}: \alpha \in D\right\}$ defined by $\phi_{x}(s)=$ $\left(m_{x_{a} x}(s)\right)_{a \in D}$ is an isomorphism of $S_{x}$ onto proj $\lim \left\{S_{x a}, m_{x a x}, \alpha \leqq \beta \in D\right\}$.

(iv) If $x, y \in X$ with $x y=x$, then $\eta_{x}\left(m_{x y}(s)\right)=x \cdot \eta_{y}(s)$ for each $s \in S_{y}$.

If $\left(T, X, S_{x}, m_{x y}, \eta_{x}\right)$ is a generalized collection and $S^{\prime}=\bigcup\left\{S_{x}: x \in X\right\}$, define $p: S^{\prime} \rightarrow X$ by $p(s)=x$ if and only if $s \in S_{x} \cdot p$ is well defined by (b)(iii).

Proposition 2.2. Let $\left(T, X, S_{x}, m_{x y}, \eta_{x}\right)$ be a generalized collection and let $S^{\prime}=\bigcup\left\{S_{x}: x \in X\right\}$. If $s, t \in S^{\prime}$, let $s \cdot t=m_{x p(s)}(s) m_{x p(t)}(t)$, where $x=p(s) p(t)$. With this multiplication, $S^{\prime}$ is an algebraic semigroup and $S^{\prime}$ is abelian if and only if each $S_{x}$ is abelian.

Proof. The proof is straightforward.

Proposition 2.3. Let the assumptions and notation be as in Proposition 2.2. Let $\Gamma$ be the basis of all open connected subsets of $T$; and, if $U \in \Gamma$ and $z \epsilon$ $U \cap X$, define $(U, z)=U$ if $z$ is isolated in $z X$, while $(U, z)=U \cap M(z)-\{z\}$ otherwise. If, then, $V \subset S_{z}$ is open, let

$$
W(U, z, V)=\left\{s \in S^{\prime}: p(s) \in(U, z), z p(s)=z \text {, and } m_{z p(s)}(s) \in V\right\} \text {. }
$$

Then, $\boldsymbol{\Upsilon}=\left\{W(U, z, V): U \in \Gamma, z \in U \cap X\right.$, and $V \subset S_{z}$ is open $\}$ is a basis for a topology on $S^{\prime}$ relative to which $S^{\prime}$ is a topological semigroup when endowed with the multiplication of Proposition 2.2.

Proof. We first show $\boldsymbol{\Upsilon}$ is a basis: Clearly $S^{\prime}=W\left(T, 0, S_{0}\right)$, and so $S^{\prime}=U r$. Suppose $s \in W\left(U_{1}, z_{1}, V_{1}\right) \cap W\left(U_{2}, z_{2}, V_{2}\right)$. For each $i$, if $z_{i}$ is isolated in $z_{i} X$, then there is $U_{i}^{\prime} \in \Gamma$ with $p(s) \in U_{i}^{\prime} \subset U_{i}$ and $z_{i} U_{i}^{\prime} \cap z_{i} X=\left\{z_{i}\right\}$. If, on the other hand, $p(s) \in\left(U_{i}, z_{i}\right)=U_{i} \cap M\left(z_{i}\right)-\left\{z_{i}\right\}$, then, since $\left(U_{i}, z_{i}\right)$ is open in $T$, there is $U_{i}^{\prime} \in \Gamma$ with $p(s) \in U_{i}^{\prime} \subset\left(U_{i}, z_{i}\right)$. If $U=\bigcap\left\{U_{i}^{\prime}: i=1,2\right\}$, clearly $p(s) \in U$ and $U$ is open and connected. If $p(s) \in X^{\prime}$, then Koch's theorem implies the existence of $z \in U \cap X$ with $z$ isolated in $z X$ and $z p(s)=z$, while if $p(s)$ $\notin X^{\prime}$, pick $z \in(U \cap[0, p(s)) \cap X)$. In either case, $p(s) \in(U, z) \subset U \subset U_{i}^{\prime} \subset$ $\left(U_{i}, z_{i}\right)$, and $z_{i} z=z_{i}$ since $z_{i} U \cap z_{i} X=\left\{z_{i}\right\}$ for $i=1$, 2. Thus, if $V=$ $\left.\bigcap_{z_{i}}^{-1}\left(V_{i}\right): i=1,2\right\}, V$ is an open subset of $S_{z}$ and $m_{z p(s)}(s) \in V$ since $m_{z_{i} p(s)}(s) \in V_{i}$ for each $i$. Then, $s \in W(U, z, V)$, and clearly $W(U, z, V) \subset$ $\bigcap\left\{W\left(U_{i}, z_{i}, V_{i}\right): i=1,2\right\}$.

The topology is Hausdorff: Let $s_{1}, s_{2} \in S^{\prime}$. If $p\left(s_{1}\right) \neq p\left(s_{2}\right)$ or $p\left(s_{1}\right)=p\left(s_{2}\right)$ $=x$ is isolated in $x X$, then it follows easily that $s_{1}$ and $s_{2}$ can be separated by disjoint open sets.

Next, we suppose $x \notin\left(X^{\prime} \cup\{0\}\right)$, and let $D=[0, x) \cap X$. Direct $D$ by $y \leqq z$ if and only if $y z=y$, and note that $\{y\}_{y \in D} \stackrel{e}{\rightarrow} x$. Then, by (d)(iii) of Definition $2.1, s_{1} \neq s_{2}$ implies there is $w \in D$ with $m_{y x}\left(s_{1}\right) \neq m_{y x}\left(s_{2}\right)$ for $w \leqq y \in D$. Since $x \in M(w)-\{w\}$, there is $U \in \Gamma$ with $x \in U \subset M(w)-\{w\}$. Let $z \in U \cap X \cap[0, x)$, 
and note that $m_{z x}\left(s_{1}\right) \neq m_{z x}\left(s_{2}\right)$. Hence, there are disjoint open subsets $V_{i}$ of $S_{z}$ with $m_{z x}\left(s_{i}\right) \in V_{i}$ for $i=1$, 2. Thus, $s_{i} \in W\left(U, z, V_{i}\right)$ for each $i$, and these sets are clearly disjoint.

Lastly, if $x \in X^{\prime}$, but $x$ is not isolated in $x X$, then $D=\{y \in x X-\{x\}: y$ is isolated in $y X\}$ is directed under $y \leqq z$ if and only if $y z=y$, and $\{y\}_{y \in D} \stackrel{e}{\rightarrow} x$ by Lemma 1.7. Again applying (d)(iii) of Definition 2.1, if $w \in D$ with $m_{y x}\left(s_{1}\right) \neq m_{y x}\left(s_{2}\right)$ for $w \leqq y \in D$, then there is $U \in \Gamma$ with $x \in U$ and $w U \cap X=\{w\}$ as $w$ is isolated in $w X$. By Koch's theorem there is $z \in U \cap D$, and if $V_{1}$ and $V_{2}$ are disjoint open subsets of $S_{z}$ with $m_{z x}\left(s_{i}\right) \in V_{i}$ for each $i$, then clearly $s_{i} \in W\left(U, z, V_{i}\right)$ for each $i$ and these sets are disjoint. This exhausts the possible cases.

Multiplication is continuous: Let $s, t \in S^{\prime}$ with $s t \in W(U, z, V)$ and let $x=$ $p(s) p(t)$. Then $x \in(U, z)$, and if $z$ is isolated in $z X$, there is $0 \in \Gamma$ with $x \in 0$ and $z 0 \cap X=\{z\}$. Let $U^{\prime}=(U, z)$ if $(U, z)=U \cap M(z)-\{z\}$, while we let $U^{\prime}=$ $0 \cap U$ if $z$ is isolated in $z X$. Now, there are $U_{i} \in \Gamma$ for $i=1,2$ with $p(s) \epsilon$ $U_{1}, p(t) \in U_{2}$, and $U_{1} U_{2} \subset U^{\prime}$. Again, we pick $z_{i} \in U_{i}$ for each $i$ so that $s \epsilon$ $W\left(U_{1}, z_{1}, S_{z_{1}}\right)$ and $t \in W\left(U_{2}, z_{2}, S_{z_{2}}\right)$, and note that $\left(U_{1}, z_{1}\right)\left(U_{2}, z_{2}\right) \subset U^{\prime}$, whence $z\left(x_{1} x_{2}\right)=z$ for any idempotents $x_{i} \in U_{i}$. Since st $\in W(U, z, V), m_{z x}(s t)$ $\epsilon V$, whence $m_{\left(z_{1} z_{2}\right) x}(s t) \in m_{z\left(z_{1} z_{2}\right)}^{-1}(V)$, and this set is open in $S_{z_{1} z_{2}}$. But,

$$
m_{\left(z_{1} z_{2}\right) x}(s t)=m_{\left(z_{1} z_{2}\right) z_{1}}\left(m_{z_{1} p(s)}(s)\right) m_{\left(z_{1} z_{2}\right) z_{2}}\left(m_{z_{2} p(t)}(t)\right),
$$

and so there are open sets $V_{i}^{\prime}$ in $S_{z_{1} z_{2}}$ for $i=1,2$ with

$$
m_{\left(z_{1} z_{2}\right) z_{1}}\left(m_{z_{1} p(s)}(s)\right) \in V_{1}^{\prime} \text { and } m_{\left(z_{1} z_{2}\right) z_{2}}\left(m_{z_{2} p(t)}(t)\right) \in V_{2}^{\prime},
$$

and $V_{1}^{\prime} V_{2}^{\prime} \subset m_{z\left(z_{1} z_{2}\right)}^{-1}(V)$. If $V_{i}=m_{\left(z_{1} z_{2}\right) z_{i}}^{-1}\left(V_{1}^{\prime}\right)$ for $i=1,2$, then $m_{z_{1} p(s)}(s)$ $\epsilon V_{1}, m_{z_{2} p(t)}(t) \in V_{2}, V_{i}$ is open in $S_{z_{i}}$ for each $i$, and so $s \in W\left(U_{1}, z_{1}, V_{1}\right)$ and $t \in W\left(U_{2}, z_{2}, V_{2}\right)$. A simple calculation now yields

$$
W\left(U_{1}, z_{1}, V_{1}\right) W\left(U_{2}, z_{2}, V_{2}\right) \subset W(U, z, V) \text {. }
$$

Lemma 2.4. Let everything be as in Propositions 2.2 and 2.3. For each $x \in X$, the topology induced on $S_{x}$ as a subset of $S^{\prime}$ is the same as the original topology on $S_{x}$.

Proof. This follows from the facts that $S_{x}$ is compact, $S^{\prime}$ is Hausdorff, and the natural embedding of $S_{x}$ into $S^{\prime}$ is continuous.

Proposition 2.5. Let everytbing be as in Propositions 2.2 and 2.3. Then, endowed with the topology of Proposition 2.3, $S^{\prime}$ is a compact space.

Proof. Let $\left\{s_{a}\right\}_{a \in D} \subset s^{\prime}$. Then, $\left\{p\left(s_{a}\right)\right\}_{a \in D} \subset X$ and $X$ is compact, whence there is $x \in X$ with $\left\{p\left(s_{a}\right)\right\}_{a \in D} \stackrel{f}{\rightarrow} x$, and by possibly picking a subnet, we may assume $\left\{p\left(s_{a}\right)\right\}_{a \in D} \stackrel{e}{\rightarrow} x$. By again picking a subnet, we have one of the following cases. 
Case 1. $x p\left(s_{\alpha}\right)=x$ for each $\alpha \in D$. Then $\left\{m_{x p\left(s_{\alpha}\right)}\left(s_{\alpha}\right)\right\}_{\alpha \in D} \subset s_{x}$, and so there is $s \in S_{x}$ with $\left\{m_{x p\left(s_{a}\right)}\left(s_{a}\right)\right\}_{a \in D} \stackrel{f}{\rightarrow} s$ in $s_{x}$. Suppose $s \in W(U, z, V)$. Then, as $\left\{p\left(s_{a}\right)\right\}_{a \in D} \stackrel{e}{\rightarrow} x$ and $x \in(U, z)$, there is $\beta_{1} \in D$ with $p\left(s_{a}\right) \in(U, z)$ for $\beta_{1} \leqq \alpha \epsilon$ $D$. Moreover, if $\beta \in D$, there is $\alpha \in D$ with $\alpha \geqq \beta_{1}, \beta$ so that $m_{x p\left(s_{a}\right)}\left(s_{a}\right) \in$ $m_{z x}^{-1}(V)$, whence $s_{a} \in W(U, z, V)$. Thus $\left\{s_{a}\right\}_{a \in D} \stackrel{f}{\rightarrow} s$ in $s^{\prime}$.

Case 2. $x_{a}=x p\left(s_{a}\right) \neq x$ for each $a \in D$. Since $\left\{p\left(s_{a}\right)\right\}_{a \in D} \stackrel{e}{\rightarrow} x,\left\{x_{a}\right\}_{a \in D} \stackrel{e}{\rightarrow}$ $x \cdot x=x$. We distinguish two subcases.

The first subcase is $x \notin\left(X^{\prime} \cup\{0\}\right)$. Let $E=[0, x) \cap X$, and direct $E$ by $y \leqq z$ if and only if $y z=y$. By (d)(iii) of Definition 2.1, $S_{x} \simeq$ proj $\lim \left\{S_{y}, m_{y z}, y \leqq z \in E\right\}$. We now define a net $\left\{t_{a}\right\}_{a \in D}$ in $\Pi\left\{S_{y}: y \in E\right\}$ by $\left(t_{a}\right)_{y}=1_{y}$ if $y p\left(s_{a}\right) \neq y$, while $\left(t_{a}\right)_{y}=m_{y p\left(s_{a}\right)}\left(s_{a}\right)$ if $y p\left(s_{a}\right)=y$. Then, as $\Pi\left\{S_{y}: y \in E\right\}$ is compact, there is $t \in \Pi\left\{S_{y}: y \in E\right\}$ with $\left\{t_{a}\right\}_{a \in D} \stackrel{f}{\rightarrow} t$, and standard arguments show $t \in \operatorname{proj} \lim \left\{S_{y}, m_{y z}, y \leqq z \in E\right\}$.

Thus, there is $s \in S_{x}$ with $\left(m_{y x}(s)\right)_{y \in E}=t$. We now show $\left\{s_{a}\right\}_{a \in D} \stackrel{f}{\rightarrow} s$.

If $s \in W(U, z, v)$, then $p(s)=x \in(U, z)$ and $z x=z$. It follows that there is an open set $U^{\prime}$ with $x \in U^{\prime} \subseteq(U, z)$ and $z U^{\prime} \cap X=\{z\}$. Since $\{y\}_{y \in E} \stackrel{e}{\rightarrow} x$, there is $w \in E$ with $y \in U^{\prime}$ for $w \leqq y \in E$. Moreover, as $\left\{p\left(s_{a}\right)\right\}_{a \in D} \stackrel{e}{\rightarrow} x$ and $x$ $\in U^{\prime} \cap M(w)-\{w\}$, there is $\beta \in D$ with $p\left(s_{\alpha}\right) \in U^{\prime} \cap M(w)-\{w\}$ for $\beta \leqq \alpha \in D$. Now, since $s \in W(U, z, V)$ and $z w=z, m_{w x}(s) \in m_{z w}^{-1}(V)$, and this set is open in $S_{w}$. Thus, as $m_{w x}(s)=t_{w}$ and $\left\{t_{a}\right\}_{a \in D} \stackrel{f}{\rightarrow} t,\left\{\alpha \in D:\left(t_{a}\right)_{w} \in m_{z w}^{-1}(V)\right\}$ is cofinal in $D$. Thus

$$
B=\left\{a \in D:\left(t_{\alpha}\right)_{w} \in m_{z w}^{-1}(V) \text { and } p\left(s_{\alpha}\right) \in U^{\prime} \cap M(w)-\{w\}\right\}
$$

is cofinal in $D$. If $a \in B$, then $p\left(s_{a}\right) \in(U, z)$ and $z p\left(s_{a}\right)=z$. Moreover, $w p\left(s_{a}\right)$ $=w$, so

$$
m_{z p\left(s_{\alpha}\right)}\left(s_{a}\right)=m_{z w}\left(m_{w p\left(s_{a}\right)}\left(s_{a}\right)\right) \in m_{z w}\left(m_{z w}^{-1}(V)\right) \subset V,
$$

whence $s_{a} \in W(U, z, V)$. Thus, $\alpha \in B$ implies $s_{\alpha} \in W(U, z, V)$, and so $\left\{s_{\alpha}\right\}_{a \in D}$ $\stackrel{f}{\rightarrow} s$.

The second subcase is $x \in X^{\prime}$. Since $\left\{x_{a}\right\}_{a \in D} \stackrel{e}{\rightarrow} x$ and $x_{a} x=x_{a} \neq x$ for each $a \in D, x$ is not isolated in $x X$. Thus, if $E=\{y \in x X-\{x\}: y$ is isolated in $y X\},\{y\}_{y \in E} \stackrel{e}{\rightarrow} x$ by Lemma 1.7 , and so $S_{x} \simeq \operatorname{proj} \lim \left\{S_{y}, m_{y z}, y \leqq z \in D\right\}$. We define the net $\left\{t_{\alpha}\right\}_{a \in D}$ exactly as in the previous subcase, and pick $t \in$ $\Pi\left\{S_{y}: y \in E\right\}$ with $\{t\}_{a \in D} \stackrel{f}{\rightarrow} t$. Again, there is $s \in S_{x}$ with $\left(m_{x y}(s)\right)_{y \in E}=t$. The proof that $\left\{s_{a}\right\}_{a \in D} \stackrel{f}{\rightarrow} s$ follows as in the previous case.

As this exhausts the possible cases, the result is established.

Lemma 2.6. Let everything be as in Propositions 2.2 and 2.3. Define $p$ : $s^{\prime} \rightarrow X$ by $p(s)=x$ if and only if $s \in S_{x}$, and define $\eta: s^{\prime} \rightarrow T$ by $\eta(s)=\eta_{p(s)}(s)$. Then, $p$ and $\eta$ are continuous surmorphisms of $S^{\prime}$ onto $X$ and $T$, respectively. 
Proof. $p$ is clearly a surmorphism, and if $U \in \Gamma$, then $p^{-1}(U)=$ $\bigcup\left\{W\left(U, z, S_{z}\right): z \in U \cap X\right\}$, and so $p$ is indeed continuous.

We now turn our attention to $\eta$. Since $\eta_{x}$ is well defined for each $x \in X$ and the $S_{x}$ 's are pairwise disjoint, $\eta$ is well defined. If $t \in T-X$, then there is $x \in X$ with $t \in[0, x]$ since $T$ has idempotent endpoints. Thus, there is $y \in X^{\prime}$ with $t \in\left[y^{\prime}, y\right]$, and so $t \in \eta_{y}\left(S_{y}\right) \subset \eta\left(S^{\prime}\right)$. If $t \in X$, then $\eta\left(1_{t}\right)=\eta_{t}\left(1_{t}\right)=t$, and so $\eta$ is surjective. That $\eta$ is a homomorphism follows essentially from (d)(iv) of Definition 2.1.

We now show $\eta$ is continuous. Let $s \in S_{x} \subset S^{\prime}$ with $\eta(s)=\eta_{x}(s) \in U \in \Gamma$. We distinguish two cases:

Case 1. $x=\eta(s)$. Then, pick $z \in U \cap X$ with $z x=z$ and $x \in(U, z)$, and let $V=\eta_{z}^{-1}(U)$. Then $V$ is an open subset of $S_{z}, \eta_{z}\left(m_{z x}(s)\right)=z \eta_{x}(s)=z \in U$, and so $m_{z x}(s) \in V$, whence $s \in W(U, z, V)$. Moreover, if $t \in W(U, z, V), p(t) \in(U, z)$ $\subset U$, and since $U$ is connected, if $r=\sup ([0, z] \cap[0, p(t)])$, then $[r, z] \cup[r, p(t)]$ $=[z, p(t)] \subset U$. If $\eta_{p(t)}(t)=p(t)$, then $\eta_{p(t)}(t) \in U$. Suppose $p(t) \in X^{\prime}$ and $\eta_{p(t)}(t) \in\left[p(t)^{0}, p(t)\right)$. Since $t \in W(U, z, V), m_{z p(t)}(t) \in V$, and so $z \eta_{p(t)}(t)=$ $\eta_{z}\left(m_{z p(t)}(t)\right) \in \eta_{z}(V) \subset U$. As $\eta_{p(t)}(t) \in\left[p(t)^{\prime}, p(t)\right), \eta_{p(t)}(t) \in[0, r] \cup[r, p(t)]$. If $\eta_{p(t)}(t) \in[0, r]$, then, since $r \in[0, z], \eta_{p(t)}(t)=z \eta_{p(t)}(t) \in U$. If $\eta_{p(t)}(t) \cdot \epsilon(r, p(t))$, then $\eta_{p(t)}(t) \in U$ as $[r, p(t)] \subset U$. In either case, $\eta(t)=\eta_{p(t)}(t) \in U$, and so $\eta(W(U, z, V)) \subset U$.

Case 2. $x \in X^{\prime}$ and $\eta_{x}(s) \in\left[x^{\prime}, x\right)$. Pick $r \in\left(\eta_{x}(s), x\right)$ and let $U_{1} \in \Gamma$ with $x \in U_{1} \subset M(r)-\{r\}$. Then, by Koch's theorem, there is $z \in U_{1} \cap x X$ with $z$ isolated in $z X$ since $x \in X^{\prime}$. If $r_{1}=\sup ([0, z] \cap[0, x])$, then, as $U_{1}$ is connected, $\left[r_{1}, z\right] \cup\left[r_{1}, x\right]=[z, x] \subset U_{1}$. Since $U_{1} \subset M(r)-\{r\}, r \in\left[0, r_{1}\right]$, and so $\left[r_{1}, x\right] \cap X$ $=\{x\}$. Moreover, $\left[r_{1}, z\right]=z\left[r_{1}, x\right]$ implies $\left[r_{1}, z\right] \cap X=\{z\}$ as translation by $z$ is a homomorphism, and so $z^{\prime}=x^{\prime}$. If $V=\eta_{z}^{-1}\left(\left[z^{\prime}, r\right) \cap U\right)$, then $V$ is open in $S_{z}$ since $\eta_{z}$ is continuous. Now, $p(s)=x \in(U, z)$ by choice of $z$, and $\eta_{z}\left(m_{z x}(s)\right)=$ $z \eta_{x}(s)=\eta_{x}(s)$ since $\eta_{x}(s) \in\left[x^{\prime}, r\right),=\left[z^{\prime}, r\right)$. Hence, as $\eta_{x}(s) \in U, m_{z x}(s) \in V$, and so $s \in W(U, z, V)$. Arguments similar to those given in Case 1 show $\eta(W(U, z, V)) \subseteq U$.

Proposition 2.7. Let everything be as in Propositions 2.2 and 2.3. If $R$ is the relation on $S^{\prime}$ whose cosets are

$$
R[s]=\left\{t \in S^{\prime}: \eta(s)=\eta(t) \text { and } m_{x p(s)}(s)=m_{x p(t)}(t)\right\},
$$

where $x=p(s) p(t)$, then $R$ is a closed congruence on $S^{\prime}$.

Proof. $R$ is clearly reflexive and symmetric. Suppose $(s, t),(t, u) \in R$. Then, $\eta(s)=\eta(t)=\eta(u)$. Let 


$$
x=p(s) p(u), \quad y=p(s) p(t) p(u), \quad z=p(s) p(t), \quad w=p(t) p(u) .
$$

We must show $m_{x p(s)}(s)=m_{x p(u)}(u)$ to show $(s, u) \in R$. Now, since $\eta(s)=\eta(t)$ $=\eta(u), \eta(s) \in \eta\left(S_{p(s)}\right) \cap \eta\left(S_{p(t)}\right) \cap \eta\left(S_{p(u)}\right)$. Moreover, $m_{x p(s)}(s), m_{x p(u)}(u) \in S_{x}$, and by (d)(iv) of Definition 2.1, $\eta\left(m_{x p(s)}(s)\right)=x \eta(s)$ and $\eta\left(m_{x p(u)}(u)\right)=x \eta(u)$.

But, as $\eta(s)=\eta(u)$,

$$
x \eta(s)=p(s) p(u) \eta(s)=p(s) p(u) \eta(u)=p(s) \eta(u)=p(s) \eta(s)=\eta(s) .
$$

Similarly, $\eta\left(m_{x p(u)}(u)\right)=\eta(u)$, and so $\eta(s) \in S_{x}$ and $\eta\left(m_{x p(s)}(s)\right)=\eta\left(m_{x p(u)}(u)\right)$. A similar argument yields the fact that $\eta(s)=\eta\left(m_{y p(s)}(s)\right)=\eta\left(m_{y p(u)}(u)\right)$, and so $\eta(s) \in \eta\left(S_{y}\right)$. Hence $\eta(s) \in \eta\left(S_{x}\right) \cap \eta\left(S_{y}\right)$, and, if $q=\inf \left(\eta\left(S_{y}\right) \cap \eta\left(S_{x}\right)\right)$ and $r=$ $\sup \left(\eta\left(S_{y}\right) \cap \eta\left(S_{x}\right)\right)$, then $\eta(s) \in[q, r]$. Moreover, as $\eta\left(m_{x p(s)}(s)\right)=\eta\left(m_{x p(u)}(u)\right)=$ $\eta(s)$, we have $m_{x p(s)}(s), m_{x p(u)}(u) \in \eta_{x}^{-1}[q, r]$, and as $\eta\left(m_{y p(s)}(s)\right)=\eta\left(m_{y p(u)}(u)\right)$ $=\eta(s), m_{y p(s)}(s), m_{y p(u)}(u) \in \eta_{y}^{-1}[q, r]$. Furthermore, using the facts that $(s, t)$ and $(t, u)$ are in $R$, it is easily shown that $m_{y x}\left(m_{x p(s)}(s)\right)=m_{y x}\left(m_{x p(u)}(u)\right)$. But, by (d)(i) and (d)(ii) of Definition 2.1, $m_{y x} \mid \eta_{x}^{-1}[q, r]$ is an injection into $\eta_{y}^{-1}[q, r]$, whence $m_{x p(s)}(s)=m_{x p(u)}(u)$. Thus $R$ is indeed transitive. A simple calculation using the fact that $\eta$ is a homomorphism and the definition of multiplication in $S^{\prime}$ yields $R$ is a congruence.

A simple argument, using the continuity of $\eta$ and $p$ and the fact that $m_{x y}(s)$ $=1_{x} \cdot s$ if $x y=x$ shows that $R$ is closed.

Definition 2.8. Let $\left(T, X, S_{x}, m_{x y}, \eta_{x}\right)$ be a generalized collection and let $S^{\prime}=\bigcup\left\{S_{x}: x \in X\right\}$ be the semigroup constructed in Propositions 2.2 and 2.3. If $R$ is the congruence on $S^{\prime}$ defined in Proposition 2.7, then $S=S^{\prime} / R$ is called the semigroup over the tree $T$ generated by the generalized collection $\left(T, X, S_{x}, m_{x y}, \eta_{x}\right)$, and is denoted $S=\delta\left(T, X, S_{x}, m_{x y}, \eta_{x}\right)$.

Lemma.2.9. If $\left(T, X, S_{x}, m_{x y}, \eta_{x}\right)$ is a generalized collection and 0 is the zero of $T$, then $S=\delta\left(T, X, S_{x}, m_{x y}, \eta_{x}\right)$ is connected if and only if $S_{0}$ is connected.

Proof. An argument utilizing the structure of $S$ similar to that given in the proof of [15, Proposition 3.6, p. 128] is straightforward.

Two of the motivations for this work were a desire to generalize the construction of the hormos to non totally-ordered semilattices, and a desire to obtain a generalization of the following theorem.

Theorem (Hofmann and Mostert [3]). Let $S$ be a compact semigroup. $\mathcal{H}$ is a congruence on $S$ and $S / \mathcal{H}$ is an I-semigroup if and only if $S=\operatorname{Horm}\left(X, S_{x}, m_{x y}\right)$ for some chainable collection $\left(X, S_{x}, m_{x y}\right)$.

The last section of this paper is devoted to. the latter of these. As to the 
former, we note that, in constructing a hormos from a chainable collection, there was no question as to how to sew the semigroups $\left\{S_{x}: x \in X\right\}$ together. However, it is easy to find examples of semilattices which can be embedded in each of the several nonisomorphic trees so as to contain the endpoints of the tree in each case, and so we include the tree $T$ in the definition of a generalized collection. We now show that the construction given in this section indeed generalizes that of the hormos.

Theorem 2.11. Let $\left(T, X, S_{x}, m_{x y}, \eta_{x}\right)$ be a generalized collection, and let 0 be the zero of $T$. If $T$ is an arc and 0 is an endpoint of $T$, then $\left(X, S_{x}, m_{x y}\right)$ is a cbainable collection and $\mathcal{S}\left(T, X, S_{x}, m_{x y}, \eta_{x}\right) \simeq \operatorname{Horm}\left(X, S_{x}, m_{x y}\right)$.

Proof. Since $T$ has idempotent endpoints and the cutpoint order agrees with the $\mathcal{H}$-order by Lemma 1.5, the endpoint other than 0 is an identity for $T$. Thus $T$ is an $I$-semigroup, and if $1=\sup T$, then 1 is the identity of $T$. If $x \in X$ with $x$ not isolated in $[0, x] \cap X$, then $b \mapsto\left(m_{y x}(b)\right)_{y<x}$ is an isomorphism of $H_{x}$ onto proj $\lim \left\{H_{y}, m_{y z}, y \leqq z<x\right\}$ after (c)(ii) and (d)(iii) of Definition 2.1, and it is now clear that $\left(X, S_{x}, m_{x y}\right)$ is a chainable collection. If $R$ is the congruence defined on $S^{\prime}=\bigcup\left\{S_{x}: x \in X\right\}$ in Proposition 2.7, and if $R^{\prime}$ is the congruence defined on $S^{\prime}$ as a chainable collection, it is routine to check $R=R^{\prime}$.

Let $\boldsymbol{r}_{1}$ be the topology on $S^{\prime}$ as a chainable collection, and let $\boldsymbol{r}_{2}$ be the topology on $S^{\prime}$ as described in Proposition 2.3. We show $i:\left(S^{\prime}, \Upsilon_{1}\right) \rightarrow\left(S^{\prime}, \Upsilon_{2}\right)$ by $i(s)=s$ is continuous. Let $s \in S_{x} \subset S^{\prime}$ with $s \in W(U, z, V)$. Then $U$ is an open connected subset of $T, p(s)=x \in(U, z)$ with $z x=z$, and $m_{z x}(s) \in V$ with $V$ an open subset of $T$. Now, $(U, z)=U$ if $z \in\left(X^{\prime} \cup\{0\}\right)$, while $(U, z)=U$ $\cap(z, 1]$ otherwise, and we let $U^{\prime}=U \cap[z, 1] \cap X$ if $z \in\left(X^{\prime} \cup\{0\}\right)$, while $U^{\prime}=$ $U \cap(z, 1] \cap X$ otherwise. Then $W\left(U^{\prime}, m_{z \mu}^{-1}(V)\right) \in \Upsilon_{1}$, where $\mu=\inf U^{\prime}$, and clearly $s \in W\left(U^{\prime}, m_{z \mu}^{-1}(V)\right)$ and $i\left(W\left(U^{\prime}, m_{z \mu}^{-1}(V)\right)\right) \subset(U, z, V)$. Thus $i$ is indeed continuous, and since $\left(S^{\prime}, \boldsymbol{r}_{1}\right)$ is compact and $\left(S^{\prime}, \boldsymbol{r}_{2}\right)$ is Hausdorff, $i$ is an isomorphism. Since $R=R^{\prime}$, the induced map $i^{*}: \operatorname{Horm}\left(X, S_{x}, m_{x y}\right) \rightarrow$ $\delta\left(T, X, S_{x}, m_{x y}, \eta_{x}\right)$ is an isomorphism, thus proving the theorem.

We note that, as a result of this theorem, the inclusion of the basis for $S_{x^{-}}$ $H_{x}$ for each $x \in X^{\prime}$ in the definition of the topology for a chainable collection is superfluous.

Proposition 2.12. Let $\left(T, X, S_{x}, m_{x y}, \eta_{x}\right)$ be a generalized collection and let $\bar{T}$ be a subtree of $T$ with idempotent endpoints. If $\bar{X}=\bar{T} \cap X$, then $(\bar{T}, \bar{X}$, $\left.S_{x}, m_{x y}, \eta_{x}\right)$ is a generalized collection and $S\left(\bar{T}, \bar{X}, S_{x}, m_{x y}, \eta_{x}\right)$ is a subsemigroup of $S\left(T, X, S_{x}, m_{x y}, \eta_{x}\right)$.

Proof. The proof is straightforward and uninteresting. 
A characterization of semigroups over trees. We are now ready to turn our attention to the main result of this work, a characterization of those compact semigroups $S$ with $\mathcal{H}$ a congruence on $S$ and $S / H$ an abelian tree with idempotent endpoints. In particular, we show that each such is $\delta\left(S / H, E(S / \mathcal{H}), S_{x}, m_{x y}, \eta_{x}\right)$, where $S_{x}$ is a suitably chosen cylindrical subsemigroup of $S$ for each $x \in E(S / \mathcal{H})$, and $m_{x y}$ is translation by $1_{x}$. Conversely, if $S=\delta\left(T, X, S_{x}, m_{x y}, \eta_{x}\right)$, then we show that $\mathcal{H}$ is a congruence on $S$ and $S / \mathcal{H} \simeq T$. It is because of this fact that we call such a semigroup a semigroup over the tree $T$. We first establish some technical lemmata we shall need for the proof of our main result, and we begin by quoting the following theorem, which is proved in [2].

Theorem 3.1.(Hofmann and Mislove[2]). Let $S$ be a compact semigroup with identity sucb that $S / M(S)$ is connected. If $s H(1) \subseteq H(1) s$ for each $s \in S$, then the identity component of the centralizer of $H(1)$ in $S$ meets $M(S)$.

Lemma 3.2. Let $S$ be an algebraic semigroup and let $e \in E(S)$. If es $\in$ Se and se $\epsilon e S$, then es $=e s e=s e$. In particular, $(e s, s e) \in \mathcal{H}$ implies es $=e s e=$ se. Finally if $\mathcal{H}$ is a congruence and $S / \mathcal{H}$ is abelian, then $E(S) \subseteq Z(S)$, the centralizer of $S$.

Proof. We prove only the last statement. If $s \in S$ and $e \in E(S)$, then (es, se) $\epsilon \mathcal{H}$ as $S / \mathcal{H}$ is abelian, and so $e s=s e$ by the first part.

Lemma 3.3. Let $S$ be a compact semigroup with $\mathcal{H}$ a congruence and $S / \mathcal{H}$ an abelian tree with idempotent endpoints. Let $X=E(S / H)$, let $x \in X^{\prime}$, and suppose $\phi_{1}: \Sigma \rightarrow \eta^{-1}\left[x^{\prime}, x\right]$ and $\phi_{2}: \Sigma \rightarrow \eta^{-1}\left[x^{\prime}, x\right]$ are homorphisms with $\eta\left(\phi_{1}(\Sigma)\right)=\eta\left(\phi_{2}(\Sigma)\right)=\left[x^{\prime}, x\right], \eta: S \rightarrow S / \mathcal{H}$ being the natural map. Then $\phi_{1}(\Sigma) \cdot H_{x}$ $=\phi_{2}(\Sigma) \cdot H_{x}$.

Proof. We show this first for $x$ isolated in $x X$.

Claim 1. There is $t \in[0, x)$ with $[0, y] \cap[0, x] \subset[0, t]$ for each $y \in x X-\{x\}$.

Proof. Suppose not, and let $D=\{y \in x X-\{x\}\}$. If, then, $t_{y}=\sup ([0, y] \cap[0, x])$ for each $y \in D$, we have $\left\{t_{y}\right\}_{y \in D} \stackrel{e}{\rightarrow} x$, where we direct $D$ by $y \leq z$ if and only if $t_{y} \in\left[0, t_{z}\right]$. Since $x X$ is compact, there is $z \in x X$ with $\{y\}_{y \in D} \stackrel{f}{\rightarrow} z$, and by possibly picking a subnet, we may assume convergence. Now $\{[0, y]\}_{y \in \underset{e}{ }} \stackrel{e}{\rightarrow}[0, z]$, and $t_{y} \in[0, y]$ for each $y \in D$, whence $x \in[0, z]$ as $\left\{t_{y}\right\}_{y \in D} \stackrel{e}{\rightarrow} x$. But $z \in x X$, and so $z x=z$. Therefore $z=x$, contradicting the fact that $x$ is isolated in $x X$, since $\{y\}_{y \in D} \stackrel{e}{\rightarrow} z$. This establishes the claim.

Let $t \in[0, x)$ with $[0, y] \cap[0, x] \subset[0, t]$ for each $y \in D$, and let $A=\eta^{-1}\left[x^{\prime}, x\right]$. Then $A$ is clearly a compact subsemigroup of $S$ with group of units $H_{x}$. Pick $s \in A$ with $\eta(s) \in(t, x]$.

Claim 2. $H_{s}(s)=s H_{x}=H_{x} s$. 
Proof. First note that $E(S) \subset Z(S)$ by Lemma 3.2. If $u \in H_{S}(s)$, then there are $a, b \in S$ with $u=s a$ and $s=u b$, whence $s=s a b$. Thus, $s=s 1_{y}$ for $1_{y} \epsilon$ $\Gamma(a b) \cap E(S)$, and so $u 1_{y}=u$ as $(s, u) \in \mathcal{H}$. If $z=x y$, then $s 1_{z}=s 1_{x} 1_{y}=s$ and $u 1_{z}=u$, whence $z \eta(s)=\eta(s)$. Now $z \in x X$ and $\eta(s)=z \eta(s) \in z[0, x]=[0, z]$, so $\eta(s) \in[0, z] \cap[0, x]$. Since $\eta(s) \in(t, x]$, we have $z=x$. Hence, $a 1_{x}, b 1_{x} \in H_{x}$ since $1_{y} \in \Gamma(a b)$ and $1_{x} 1_{y}=1_{x}$. Moreover, $s\left(a 1_{x}\right)=(s a) l_{x}=u 1_{x}=u$, and so $H_{s}(s) \subset s H_{x}$.

If $a \in H_{x}$, then $\eta(s a)=\eta(s) \eta(a)=\eta(s) x=\eta(s)$, and so $s H_{x} C H_{s}(s)$. Therefore $H_{s}(s)$ $=s H_{x}$, and a similar argument shows $H_{S}(s)=H_{x} s$, proving the claim.

Now, $A=H_{x} \cup \eta^{-1}\left[x^{\prime}, x\right)$, and $\eta\left(H_{x}\right) \cap \eta\left(\eta^{-1}\left[x^{\prime}, x\right)\right)=\square$. Hence, since $H_{x}$ is closed in $A$ and $\eta$ is a closed map, $H_{x}$ is not open in $A$ as $\left[x^{\prime}, x\right]$ is connected.

It follows that there is a one parameter semigroup in $A$ containing $1_{x}$ whose closure meets $M(A)$, and from this that $A / M(A)$ is connected. This, along with Claims 1 and 2 imply that $A$ satisfies the hypotheses of Theorem 3.1. Therefore, the identity component of the centralizer of $H\left(1_{x}\right)$ in $A, C$, meets $M(A)$, and since $1_{x}$ is isolated in $C$, there is a one-parameter semigroup in $C$ containing $1_{x}$ whose closure meets $M(C) \subseteq M(A)$. Thus there is a homomorphism $\phi: \Sigma \times H_{x} \rightarrow$ $A$ with $\phi((0,0), b)=b$ for each $b \in H_{x}$ and $\phi\left(\Sigma \times H_{x}\right) \not \subset H_{x}[3$, p. 87, 2.3]. Hence, $\eta\left(\phi\left(\Sigma \times H_{x}\right)\right)=\left[x^{\prime}, x\right]$, and it now suffices to show $\phi\left(\Sigma \times H_{x}\right)=\phi^{\prime}(\Sigma) \cdot H_{x}$ for any $\phi^{\prime}: \Sigma \rightarrow A$ with $\eta\left(\phi^{\prime}(\Sigma)\right)=\left[x^{\prime}, x\right]$.

Let $s \in \phi^{\prime}(\Sigma)$ with $\eta(s) \in(t, x]$. Then there is $s^{\prime} \in \phi\left(\Sigma \times H_{x}\right)$ with $\eta\left(s^{\prime}\right)$ $=\eta(s)$, and so there is $b \in H_{x}$ with $s=s^{\prime} b$ by Claim 2. Hence $s=s^{\prime} b \in$ $\phi\left(\Sigma \times H_{x}\right) H_{x}=\phi\left(\Sigma \times H_{x}\right)$, and so $\phi^{\prime}(\Sigma) \subset \phi\left(\Sigma \times H_{x}\right)$ since $\phi^{\prime}(\Sigma)$ is generated by $\left\{s \in \phi^{\prime}(\Sigma): \eta(s) \in(t, x]\right\}$. Therefore, $\phi^{\prime}(\Sigma) \cdot H_{x} \subset \phi\left(\Sigma \times H_{x}\right) \cdot H_{x}=\phi\left(\Sigma \times H_{x}\right)$.

Now, there is $r \in \mathbf{H}$ with $r>0$ and $\phi\left((p, s(p)), 1_{x}\right) \in \eta^{-1}(t, x]$ for $r>p \in \mathbf{H}$, and so $\eta\left(\phi\left((p, s(p)), 1_{x}\right)\right)=\eta(s)$ for some $s \in \phi^{\prime}(\Sigma)$. Moreover, by Claim 2, there is $b_{p} \in H_{x}$ with $\phi\left((p, s(p)), 1_{x}\right)=s b_{p}$, whence

$$
\phi\left(\{(p, s(p)): p<r\} \times\left\{1_{x}\right\}\right) \subset \phi^{\prime}(\Sigma) \cdot H_{x} \text {. }
$$

But $\Sigma \times\left\{1_{x}\right\}=\left\langle\{(p, s(p)): p<r\} \times\left\{1_{x}\right\}\right\rangle^{*}$, and so

$$
\phi\left(\Sigma \times\left\{1_{x}\right\}\right)=\phi\left(\left\langle\{(p, s(p)): p<r\} \times\left\{1_{x}\right\}\right\rangle^{*}\right) \subset \phi^{\prime}(\Sigma) \cdot H_{x^{\bullet}}
$$

Finally, $\phi\left(\Sigma \times H_{x}\right)=\phi\left(\Sigma \times\left\{1_{x}\right\}\right) \cdot H_{x} \subset \phi^{\prime}(\Sigma) \cdot H_{x} \cdot H_{x}=\phi^{\prime}(\Sigma) \cdot H_{x^{\circ}}$ This esblishes the desired result in the case that $x$ is isolated in $x X$.

Now, suppose $x \in X^{\prime}$ and $x$ is not isolated in $x X$. If $D=\{y \in x X: y$ : isolated in $y X\}$, then $D$ is directed under $y \leqq z$ if and only if $y z=y$ and $\{y\}_{y \in D} \stackrel{e}{\rightarrow} x$ by Lemma 1.7. Moreover, by possibly picking a residual subset of $D$, we may assume $y^{\prime}=x^{\prime}$ for each $y \in D$ by Proposition 1.6. Now, if $\left\{H_{a}\right\}_{a \in E}$ 
is a universal subnet of $\left\{H_{y}\right\}_{y \in D}\left[5\right.$, p. 81], then $\left\{H_{a}\right\}_{a \in E}$ converges to some subsemigroup $H$ of $S_{i}$ Moreover, as $\eta: S \rightarrow S / \mathcal{H}$ is continuous, if $\left\{h_{y}\right\}_{y} \in D \stackrel{f}{\rightarrow} b \in S$, then $\left\{\eta\left(b_{y}\right)\right\}_{y \in D} \stackrel{f}{\rightarrow} \eta(b)$, and so $\eta(b)=x$ since $\eta\left(b_{y}\right)=y$ for each $y \in D$ and $\{y\}_{y \in D} \rightarrow x$. Thus $b \in H_{x}$, and we have $H \subset H_{x}$. But $H_{x} \subset H$ since $1_{y} H_{x} \subset H_{y}$ for each $y \in D$, and so $H=H_{x}$.

Now, let $\phi_{1}: \Sigma \rightarrow \eta^{-1}\left[x^{\prime}, x\right]$ and $\phi_{2}: \Sigma \rightarrow \eta^{-1}\left[x^{\prime}, x\right]$ be homomorphisms with $\eta\left(\phi_{1}(\Sigma)\right)=\eta\left(\phi_{2}(\Sigma)\right)=\left[x^{\prime}, x\right]$. If $y \in D$, since $E(S) \subset Z(S)$ by Lemma 3.2, translation by $1_{y}$ is a homomorphism, and moreover,

$$
\eta\left(1_{y} \cdot \eta^{-1}\left[x^{\prime}, x\right]\right)=\eta\left(1_{y}\right) \cdot\left[x^{\prime}, x\right]=\left[\eta\left(1_{y}\right) x^{\prime}, \eta\left(1_{y}\right) x\right]=\left[\eta\left(1_{y}\right)^{\prime}, \eta\left(1_{y}\right)\right],
$$

whence $1_{y} \cdot \eta^{-1}\left[x^{\prime}, x\right] \subset \eta^{-1}\left[\eta(1)^{\prime}, \eta\left(1_{y}\right)\right]$. Hence, if $y \in D$, then $\phi_{i y}: \Sigma \rightarrow$ $\eta^{-1}\left[\eta\left(1_{y}\right)^{1}, \eta\left(1_{y}\right)\right]$ by $\phi_{i y}(s)=1_{y} \cdot \phi(s), i=1,2$, are homormophisms and $\eta\left(\phi_{i y}(\Sigma)\right)=\left[\eta\left(1_{y}\right)^{\prime}, \eta\left(1_{y}\right)\right]$ for each $i$. Thus, $\phi_{1 \dot{y}}(\Sigma) \cdot H_{y}=\phi_{2 y}(\Sigma) \cdot H_{y}$ by the first part of this proof. Therefore,

$$
\begin{aligned}
\phi_{1}(\Sigma) \cdot H_{x} & =\phi_{1}(\Sigma) \cdot \lim H_{y}=\lim \phi_{1}(\Sigma) \cdot H_{y}=\lim \phi_{1 y}(\Sigma) \cdot H_{y} \\
& =\lim \phi_{2 y}(\Sigma) \cdot H_{y}=\phi_{2}(\Sigma) \cdot H_{x} \cdot
\end{aligned}
$$

This concludes the proof of the lemma.

Main theorem. Let $S$ be a compact semigroup. $H$ is a congruence on $S$ and $S / H$ is an abelian tree with idempotent endpoints if and only if $\mathcal{S}\left(T, X, S_{x}, m_{x y}, \eta_{x}\right)$ for some generalized collection $\left(T, X, S_{x}, m_{x y}, \eta_{x}\right)$.

Proof. We first establish necessity. Suppose $S$ is a compact semigroup with $\mathcal{H}$ a congruence on $S$ and $S \mathcal{H}$ an abelian tree with idempotent endpoints. We now construct a generalized collection $\left(T, X, S_{x}, m_{x y}, \eta_{x}\right)$.

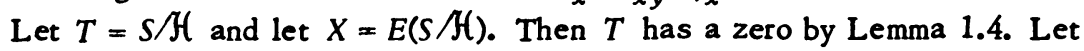
$x \in X^{\prime}$ and consider $\eta^{-1}\left[x^{\prime}, x\right], \eta: S \rightarrow S / \mathcal{H}$ being the natural map. If $x$ is isolated in $x X$, then, as in the proof of Lemma 3.4, there is a homomorphism $\phi: \Sigma \times$ $H_{x} \rightarrow \eta^{-1}\left[x^{\prime}, x\right]$ with $\phi((0,0), b)=b$ for each $b \in H_{x}$ and $\eta(\phi(\Sigma))=\left[x^{\prime}, x\right]$. If $\left(r, r^{\prime}\right) \in \Sigma$ and $b \in H_{x}$, then

$$
\phi\left(\left(r, r^{\prime}\right), 1_{x}\right) b=\phi\left(\left(r, r^{\prime}\right), b\right)=\phi((0,0), b) \phi\left(\left(r, r^{\prime}\right), 1_{x}\right)=h \phi\left(\left(r, r^{\prime}\right), 1_{x}\right),
$$

and so $\phi\left(\Sigma \times\left\{1_{x}\right\}\right) \subset Z\left(H_{x}\right)$. Thus, if $C_{x}$, is the identity component of the centralizer of $H_{x}$ in $\eta^{-1}\left[x^{\prime}, x\right]$, then $C_{x}$ contain $1_{x^{\prime}}$.

If, on the other hand, $x$ is not isolated in $x X$, then arguments similar to those given in the consideration of the analogous part of the proof of Lemma 3.3 show that $1_{x^{\prime}} \in C$, the identity component of the centralizer of $H_{x}$ in $\eta^{-1}\left[x^{\prime}, x\right]$.

If now, $H$ is the group of units of $C$, then $H$ is closed in $C, H \neq C$, and, as $C$ is connected, $H$ is not open in $C$. Since $C \subset \eta^{-1}\left[x^{\prime}, x\right], 1_{x}$ is isolated in the 
set of idempotents of $C$, and so there is a one-parameter semigroup $f: H \rightarrow C$ with $f(0)=1_{x}$ and $f(\mathbf{H}) \not \subset H$. Since $C$ is a subset of the centralizer of $H_{x}$ in $\eta^{-1}\left[x^{\prime}, x\right]$, there is a homomorphism $\phi: \Sigma \times H_{x} \rightarrow \eta^{-1}\left[x^{\prime}, x\right]$ with $\phi((0,0), b)=$ $b$ for each $b \in \mathbf{H}_{x}$ and $\eta\left(\phi\left(\Sigma \times H_{x}\right)\right)=\left[x^{\prime}, x\right][3$, p. 87, 2.3].

Thus, in either case, if $x \in X^{\prime}$, there is a homomorphism $\phi: \Sigma \times H_{x} \rightarrow$ $\eta^{-1}\left[x^{\prime}, x\right]$ with $\phi((0,0), b)=b$ for each $b \in H_{x}$ and $\eta\left(\phi\left(\Sigma x H_{x}\right)\right)=\left[x^{\prime}, x\right]$. Pick one such, and let $S_{x}=\phi\left(\Sigma \times H_{x}\right)$. If $x \notin X^{\prime}$, let $S_{x}=H_{x}$. If $x, y \in X$ with $x y=$ $x$, let $m_{x y}: S_{y} \rightarrow S_{x}$ be defined by $m_{x y}(s)=1_{x} s$. Since $S / \mathcal{H}$ is an abelian tree with idempotent endpoints, $E(S) \subset Z(S)$ by Lemma 3.2, and so $m_{x y}$ is a homomorphism. If $y \notin X^{\prime}$, then $S_{y}=H_{y}$ and, if $b \in H_{y}$

$$
m_{x y}(b) m_{x y}\left(b^{-1}\right)=\left(1_{x} b\right)\left(1_{x} b^{-1}\right)=1_{x}\left(b b^{-1}\right)=1_{x y} 1_{x}=1_{x} \text {, }
$$

whence $m_{x y}\left(S_{y}\right) \subset H_{x} \subset S_{x^{-}}$. Suppose $y \in X^{\prime}$. If $x \notin X^{\prime}$, then $x\left[y^{\prime}, y\right] \subset[0, x]$ and, as translation by $x$ is a homomorphism, $x\left[y^{\prime}, y\right] \cap X=\left\{x y^{\prime}, x\right\}$, whence $x y^{\prime}$ $=x$. Hence if $s \in S_{y}$,

$$
\eta\left(m_{x y}(s)\right)=\eta\left(1_{x} s\right)=\eta\left(1_{x}\right) \eta(s)=x \eta(s)=x,
$$

and so $m_{x y}(s) \in H_{x} \subset S_{x^{\circ}}$ Suppose $x \in X^{\prime}$. Then $x\left[y^{\prime}, y\right] \subset[0, x]$, and $x\left[y^{\prime}, y\right]$ $\cap X=\left\{x y^{\prime}, x\right\}$ as above. Thus $x y^{\prime}=x$ or $x y^{\prime}=x^{\prime}$, and so, if $s \in S_{y} \subset \eta^{-1}\left[y^{\prime}, y\right]$, then $\eta\left(m_{x y}(s)\right)=\eta\left(1_{x} s\right)=x \eta(s) \in\left[x^{\prime}, x\right]$, whence $m_{x y}\left(S_{y}\right) \subset \eta^{-1}\left[x^{\prime}, x\right]$. Therefore, if $S_{y}=\phi\left(\Sigma \times H_{y}\right)$, then $m_{x y} \circ \phi \mid\left(\Sigma \times\left\{1_{y}\right\}\right)$ is a homomorphism of $\Sigma \times\left\{1_{y}\right\}$ into $\eta^{-1}\left[x^{\prime}, x\right]$, and so $m_{x y}\left(\phi\left(\Sigma x\left\{1_{y}\right\}\right)\right) \subset S_{x}$ by Lemma 3.3. Thus

$$
\begin{aligned}
m_{x y}\left(S_{y}\right) & =m_{x y}\left(\phi\left(\Sigma \times H_{y}\right)\right)=m_{x y}\left(\phi\left(\Sigma \times\left\{1_{y}\right\}\right) H_{y}\right) \\
& =m_{x y}\left(\phi\left(\Sigma \times\left\{1_{y}\right\}\right) m_{x y}\left(H_{y}\right) S_{x} \cdot H_{x}=S_{x},\right.
\end{aligned}
$$

the containment following from the obvious fact that $m_{x y}\left(H_{y}\right) \subset H_{x^{*}}$

In any case, $m_{x y}\left(S_{y}\right) \subset S_{x}$, and $m_{x y}\left(S_{y}\right) \subset H_{x}$ if $x \in[0, y)$. In particular, if $y \in X^{\prime}$ and $x=y^{\prime}, M_{y} \subset H_{x}$, and so $m_{x y} \mid M_{y}$ is the identity map, and hence it is an injection. If $x \in X$, let $\eta_{x}=\eta \mid S_{x}$. If $x, y \in X$ with $x y=x$ and $s \in S_{y}$, $\eta_{x}\left(m_{x y}(s)\right)=\eta\left(1_{x} s\right)=x \eta(s)=x \eta_{y}(s)$. As $s_{x}$ is cylindrical, $\mathcal{H}_{s_{x}}=\mathcal{H}_{s} \cap\left(s_{x} \times s_{x}\right)$ [9, Lemma 2.4], and so (b)(ii) of 2.1 holds. Moreover, if $x y=x$ and $y z=y$, then $m_{x y} \circ m_{y z}=m_{x z}$ is clear as $1_{x} 1_{y}=1_{x}$.

Suppose $x, y \in X^{\prime}, x y=x$, and $x^{\prime}=y^{\prime}$. Then, if $t=\sup \left(\left[x^{\prime}, x\right] \cap\left[y^{\prime}, y\right]\right)$ and if $s \in \eta_{y}^{-1}\left[y^{\prime}, t\right]$, then $\eta(s) \in\left[x^{\prime}, t\right]$, and so $x \eta(s)=\eta(s)$ by Lemma 1.5. Hence $m_{x y}(s)=1_{x} s=s$, and so $m_{x y} \mid \eta_{y}^{-1}\left[y^{\prime}, t\right]$ is an injection into $\eta_{x}^{-1}\left[x^{\prime}, t\right]$.

We have shown that $\left(T, X, S_{x}, m_{x y}, \eta_{x}\right)$ satisfies all the conditions of Definition 2.1 except (d)(iii) and (b)(iii). We consider these in that order.

Suppose $\left\{x_{a}\right\}_{a \in D} \subset X$ such that $\left\{x_{a}\right\}_{a \in D} \stackrel{e}{\rightarrow} x \in X$ with $x_{a} x=x_{a}$ for each $a \in D$ and $\alpha \leqq \beta$ implies $x_{a} x_{\beta}=x_{\alpha}$ Let $\phi_{x}: S_{x} \rightarrow \Pi\left\{S_{x}: a \in D\right\}$ be defined by $\phi_{x}(s)=\left(m_{x_{a} x}(s)\right)_{a \in D^{*}}$ 
$\phi_{x}$ is a homomorphism as each $m_{x_{a x}}$ is, and clearly $\phi_{x}\left(S_{x}\right) \subset$ proj $\lim \left\{S_{x_{a}}, m_{x_{a} \beta^{*}}, a \leqq \beta \in D\right\}$. Since $\left\{1_{x_{a}}\right\}_{a \in D} \stackrel{e}{\rightarrow} 1_{x}$, if $\phi_{x}(s)=\phi_{x}(t)$, then

$$
s=\left(\lim 1_{x_{a}}\right) s=\lim 1_{x_{a}} s=\lim \left(\phi_{x_{a}}(s)\right)_{a}=\lim \left(\phi_{x_{a}}(t)\right)_{a}=t,
$$

whence $\phi_{x}$ is one-to-one. To show $\phi_{x}$ is surjective, fix $\left(s_{a}\right)_{\alpha \in D} \epsilon$ proj $\lim \left\{S_{x_{a}}, m_{x_{\alpha \times \beta} \beta^{\circ}}, a \leqq \beta \in D\right\}$. We distinguish two cases.

Case 1. $x \notin X^{\prime}$, in which case $S_{x}=H_{x}$. By possibly picking a subnet, either $x_{a} \in X^{\prime}$ for each $a \in D$ or $x_{a} \not X^{\prime}$ for each $a \notin D$. In the former case, if $U$ is a connected open subset of $T$ containing $x$, then there is $y \in U \cap[0, x) \cap X$ so $x \in U \cap M(y)-\{y\}$. Thus there. is $\beta \in D$ with $x_{a} \in U \cap M(y)-\{y\}$ for $\beta \leqq a \in D$, whence $y \in\left[0, x_{a}\right]$ for $\beta \leqq \alpha \in D$. As $y, x_{\alpha} \in U$ and $U$ is connected, $\left[y_{,} x_{a}\right] \subset U$ for $\beta \leqq \alpha \in D$, and, since $y \in\left[0, x_{a}\right] \cap X, x_{a}^{\prime} \in U$ for $\beta \leqq \alpha \in D$. Thus $\left\{x_{a}^{\prime}\right\}_{a \in D} \stackrel{e}{\rightarrow} x$, and therefore, since $x_{\alpha}^{\prime} \leqq \eta\left(s_{a}\right) \leqq x_{a}$ for each $\alpha \in D$, we have $\left\{\eta\left(s_{a}\right)\right\}_{a \in D} \stackrel{e}{\rightarrow} x$ if $x_{a} \in X^{\prime}$ for each $\alpha \in D$, this fact being obvious if $x_{a} \notin D$.

As $S$ is compact, there is $s \in S$ with $\left\{s_{a}\right\}_{a \in D} \stackrel{f}{\rightarrow} s$, and, by the above, $s \epsilon$ $s_{x^{*}}$ Standard arguments now show $1_{x_{a}} \cdot s=s_{a}$ for each $a \in D$, so $\phi(s)=\left(s_{a}\right)_{a \in D^{*}}$

Case $2 x \in X^{\prime}$. According to Lemma 1.6, we may assume $x_{\alpha} \in X^{\prime}$ and $x_{a}^{\prime}=x^{\prime}$ for each $a \in D$. Let $S_{x}=\phi\left(\Sigma \times H_{x}\right)$ with $\phi((0,0), b)=b$ for each $b \in H_{x^{*}}$ Then, by Lemma 3.3, $S_{x_{a}}=\left[\left(m_{x_{a} x} \circ \phi\right)\left(\Sigma \times\left\{1_{x}\right\}\right)\right] \cdot H_{x_{a}}$, and so, for each $a \in D$, there are $t_{a} \in \phi\left(\Sigma \times\left\{1_{x}\right)\right.$ and $h_{a} \in H_{x_{a}}$ with $s_{\alpha}=m_{x_{a} x}\left(t_{\alpha}\right) h_{\alpha}$. There is $t \in \phi\left(\Sigma \times\left\{1_{x}\right\}\right)$ $\phi\left(\Sigma \times\left\{1_{x}\right)\right.$ with $\left\{t_{a}\right\}_{a \in D} \stackrel{f}{\rightarrow} t$, and so there is a subnet $\left\{t_{\beta}\right\}_{\beta \in E}$ with $\left\{t_{\beta}\right\}_{\beta \in E} \stackrel{e}{\rightarrow} t$. Since $S$ is compact, there is $b \in S$ with $\left\{b_{\beta}\right\}_{\beta \in E} \stackrel{f}{\rightarrow} b$, and, since $\eta: S \rightarrow S / \mathcal{H}$ is continuous and $\left\{x_{\beta}\right\}_{\beta \in E} \stackrel{e}{\rightarrow} x, b \in H_{x}$. By possibly picking another subnet, we may assume $\left\{b_{\beta}\right\}_{\beta \in E} \stackrel{e}{\rightarrow} b$. Now, $s=t b \in \phi\left(\Sigma \times\left\{1_{x}\right\}\right) \cdot H_{x}=S_{x}$, and again standard arguments show $\phi(s)=\left(s_{a}\right)_{a \in D^{\circ}}$.

Thus, property (d)(iii) of Definition 2.1 is fulfilled, and, to accomplish (b)(iii), we make the following inessential changes: for $x \in X$, let $T_{x}=\{x\} \times S_{x}$, and, if $x, y \in X$ with $x y=x$, define $m_{x y}^{\prime}: T_{y} \rightarrow T_{x}$ by $m_{x y}^{\prime}(y, s)=\left(x, m_{x y}(s)\right)$. Let $\eta_{x}^{\prime}: T_{x} \rightarrow\left[x^{\prime}, x\right]$ be defined by $\eta_{x}^{\prime}(x, s)=\eta_{x}(s)$. Clearly $\left(T, X, T_{x}, m_{x}^{\prime}, \eta_{x}^{\prime}\right)$ is a generalized collection.

Let $\bar{S}=S\left(T, X, T_{x}, m_{x}^{\prime}, \eta_{x}^{\prime}\right)$. We show $\bar{S} \simeq S$. Let $S^{\prime}=\bigcup\left\{T_{x}: x \in X\right\}$ be the semigroup constructed in Propositions 2.2 and 2.3, and define $f: S^{\prime} \rightarrow S$ by $f(x, s)=s$ for each $(x, s) \in S^{\prime}$. We will show that $f$ is a continuous surmorphism and that $\rho_{f}=R$, the congruence defined in Proposition 2.7.

Clearly $f$ is well defined. Let $s \in S$. If $\eta(s)=x \in X$, then $s \in H_{x} \subset s_{x}$, and so $(x, s) \in T_{x}$ and $f(x, s)=s$. Suppose $\eta(s) \in\left(x^{\prime}, x\right)$ for some $x \in X^{\prime}$. Then, since $\eta\left(S_{x}\right)=\left[x^{\prime}, x\right]$, there is $t \in S_{x}$ with $\eta(t)=\eta(s)$, whence $(s, t) \in \mathcal{H}_{S}$, and so there are $a, b \in S$ with $s a=t$ and $t b=s$. Then, $s=s a b$, and, therefore $s=s 1_{y}$ 
where $1_{y} \in \Gamma(a b)$. Now, $a 1_{y}, b 1_{y} \in S 1_{y}$ and $\left\{\left(a 1_{y} b 1_{y}\right)^{n}\right\}_{n \in \omega} \stackrel{f}{\rightarrow} 1_{y}$, whence $a 1_{y}$, $b \mathrm{l}_{y} \in H_{y}$. If $z=x y$, then

$$
m_{z y}\left(S_{x}\right) \subset S_{z} \text {, and } m_{z x}(t) m_{z y}\left(b 1_{y}\right)=\left(1_{z} t\right)\left(1_{z} b 1_{y}\right)=1_{z} t b=1_{z} s=s \text {. }
$$

Thus, $s \in S_{z}$, and so $(z, s) \in T_{z}$ and $f(z, s)=s$. Therefore, $f$ is surjective.

To show the continuity of $f$, let $\left\{\left(x_{\alpha}, s_{\alpha}\right)\right\}_{a \in D} \subset S^{\prime}$ with $\left\{\left(x_{\alpha}, s_{\alpha}\right)\right\}_{a \in p} \stackrel{e}{\rightarrow}$ $(x, s)$. Then $\left\{s_{a}\right\}_{a \in D}$ is a net in $S$, and so there is $t \in S$ with $\{s\}_{\alpha \in D} \stackrel{P}{\rightarrow} t$. By picking a subnet, we may assume $\left\{s_{a}\right\}_{a \in D} \stackrel{e}{\rightarrow} t$. If $y_{a}=x x_{a}$ for each $a \in D$, then, by possibly picking a subnet, we may assume that either $y_{a} \neq x$ for each $a \in D$ or $y_{a}=x$ for each $\alpha \in D$. In the first case, $x$ is not isolated in $x X$, and as in the last case of the proof of Proposition 2.5, we pick a net $\{y\}_{y \in E} \subset x X$ with $y z=y$ if $y \leqq z$ and $\{y\}_{y \in E} \stackrel{e}{\rightarrow} x$. Moreover, as is shown in that proof $\left\{a \in D: y y_{a}=y\right\}$ is residual in $D$ for each $y \in E$. If $y_{x}=x$ for each $a \in D$, we let $\{y\}_{y \in E}$ be the constant net $\{x\}$. Fix $y \in E$ and let $\beta \in D$ with $y y_{a}=y$ for $\beta \leqq \alpha \in D$. Then, $\left\{m_{y x}\left(s_{a}\right)\right\}_{\beta \leqq a} \subset S_{y}$ and $\left\{m_{y x_{a}}\left(s_{\alpha}\right)\right\}_{\beta \leqq a} \stackrel{e}{\rightarrow} m_{y x}(s)$ in $s_{y}$ as a subset of $s^{\prime}$. Moreover, $\left\{1_{y} s_{a}\right\}_{\beta \leqq a} \stackrel{e}{\rightarrow} 1_{y} t$ in $S$ as multiplication in $S$ is continuous. But, $m_{y x_{a}}\left(s_{a}\right)$ $=1_{y} s_{a}$ for each $\beta \leqq \alpha \in D$, and since $S_{y}$ is a closed subset of $S, 1_{y} t \in S_{y}$. But, by Lemma 2.4 , the topology on $S_{y}$ as a subset of $S^{\prime}$ is the same as the topology on $S_{y}$ as a subset of $S$, and so $m_{y x}(s)=1_{y} t$. Since $y \in E$ is arbitrary and $1_{y} s=$ $m_{y x}(s)$ for each $y \in E, s=1_{x} s=\left(\lim 1_{y}\right) s=\lim 1_{y} s=\lim 1_{y} t=t$, the last equality following from the fact that $\left\{s_{a}\right\}_{a \in D} \stackrel{e}{\rightarrow} t$ and $\left\{1_{x_{a}}\right\}_{a \in D} \stackrel{e}{\rightarrow} 1_{x^{*}}$ Thus, $\left\{f\left(x_{a}, s_{a}\right)\right\}_{\alpha \in D} \stackrel{e}{\rightarrow} f(x, s)$, and $f$ is indeed continuous.

Suppose $(x, s),(y, t) \in S^{\prime}$. Then, if $z=x y$,

$$
\begin{aligned}
f((x, s)(y, t)) & =f\left(z, m_{z x}(s) m_{z y}(t)\right)=f\left(z,\left(1_{z} s\right)\left(1_{z} t\right)\right) \\
& =1_{z} s t=1_{x} 1_{y} s t=1_{x} s 1_{y} t=s t=f(x, s) f(y, t),
\end{aligned}
$$

whence $f$ is a homomorphism.

We now show $\rho_{f}=R$. If $f(x, s)=f(y, t)$, then $s=t$, and so $\eta(s)=\eta(t)$. Moreover, if $z=x y$, then

$$
m_{z x}^{\prime}(x, s)=\left(z, m_{z x}(s)\right)=\left(z, 1_{z} s\right)=\left(z, 1_{z} t\right)=\left(z, m_{z y}(t)\right)=m_{z y}^{\prime}(t) .
$$

Therefore, since $\eta_{x}^{\prime}(x, s)=\eta(s)=\eta(t)=\eta_{y}^{\prime}(y, t),((x, s),(y, t)) \in R$, and so $\rho_{f} \subset R$. If, conversely, $((x, s),(y, t)) \in R$, then $\eta(s)=\eta_{x}^{\prime}(x, s)=\eta_{y}^{\prime}(y, t)=\eta(t)$. But, $1_{x} s=s$ and $1_{y} t=t$ as $s \in S_{x}$ and $t \in S_{y}$, and so $1_{y} s=s$ and $1_{x} t=t$ since $(s, t)$. $\in \mathcal{H}$. Now, if $z=x y$, then $s=1_{x} 1^{s}=1_{z} s=m_{z x}(s)=m_{z y}(t)=1_{z} t=1_{x} 1_{y} t=t$, and we have $f(x, s)=s=t=f(y, t)$, whence $((x, s),(y, t)) \in \rho_{f}$. Therefore $R \subset \rho_{f}$, and
so $R=\rho_{f}$ 
We therefore have the following commutative diagram:

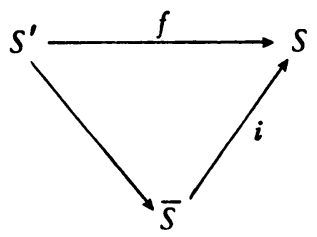

and $i$ is an isomorphism since $R=\rho_{f}$. This concludes the proof of the necessity.

To show sufficiency, suppose $S=S\left(T, X, S_{x}, m_{x y}, \eta_{x}\right)$ is the semigroup over the tree $T$ generated by the generalized collection $\left(T, X, S_{x}, m_{x y}, \eta_{x}\right)$. First, to see that $\mathcal{H}$ is a congruence, let $(a, b) \in \mathcal{H}$ and let $c \in S$. If $a \in S_{x}$ and $b \in S_{y}$, then $1_{x} a=1_{y} a=a$ and $1_{x} b=1_{y} b=b$. Thus, $a, b \in S_{z}$, where $z=x y$, and so $(a, b) \in \mathcal{H}_{S_{z}}$ [9, Lemma 2.4]. If $c \in S_{w}$ and $v=z w$, then $\left(m_{v z}(a), m_{v z}(b)\right) \in \mathcal{H}_{S_{v}}$ as $m_{v z}$ is a homomorphism, and, since $S_{v}$ is cylindrical, $\mathcal{H}_{S_{v}}$ is a congruence, whence $\left(m_{v z}(a) m_{v w}(c), m_{v z}(b) m_{v w}(c)\right) \in \mathcal{H}_{S_{v}}$. But, $a c=m_{v w}(a) m_{v w}(c)$ and $b c=$ $m_{v z}(b) m_{v w}(c)$ and $\mathcal{H}_{s_{v}} \subset \mathcal{H}$ and so $(a c, b c) \in \mathcal{H}$. Similarly, $(c a, c b) \in \mathcal{H}$, and so $\mathcal{H}$ is indeed a congruence on $S$.

Let $\eta: S^{\prime}=\bigcup\left\{S_{x}: x \in X\right\} \rightarrow T$ be defined by $\eta(s)=\eta_{x}(s)$, where $s \in S_{x}$, and let $\phi: S^{\prime} \rightarrow S=S^{\prime} / R$ be the natural map, $R$ being the congruence defined in Proposition 2.7, and, lastly, let $\nu: S \rightarrow S \mathcal{H}$ be the natural map. We show $S / \mathcal{H} \simeq T$ by showing that $\rho_{\eta}=\rho_{\nu \circ \phi}$, thus establishing that $S \mathcal{H}$ is an abelian tree with idempotent endpoints.

If $(s, t) \in \rho_{\eta}$, then $\eta_{p(s)}(s)=\eta_{p(t)}(t)$, where $p: s^{\prime} \rightarrow X$ is defined by $p(s)$ $=x$ if and only if $s \in S_{x}$. If $x=p(s) p(t)$, then

$$
\eta_{x}\left(m_{x p(s)}(s)\right)=x \eta_{p(s)}(s)=x \eta_{p(t)}(t)=\eta_{x}\left(m_{x p(t)}(t)\right),
$$

and so $\nu\left(\phi\left(m_{x p(s)}(s)\right)\right)=\nu\left(\phi\left(m_{x p(t)}(t)\right)\right)$ as $\eta_{x}$ is the $\mathcal{H}$-class map on $S_{x}$. Thus, $\left(m_{x p(s)}(s), m_{x p(t)}(t)\right) \in \rho_{\nu_{0} \phi}$, and, furthermore,

$$
\begin{aligned}
\eta\left(m_{x p(s)}(s)\right) & =x \eta_{p(s)}(s)=p(t) p(s) \eta_{p(s)}(s)=p(t) \eta_{p(s)}(s)=p(t) \eta_{p(t)}(t) \\
& =\eta_{p(t)}(t)=\eta_{p(s)}(s),
\end{aligned}
$$

and so $\left(s, m_{x p(s)}(s)\right) \in R$. Similarly, $\left(m_{x p(t)}(t), t\right) \in R$, and therefore, $\left(s, m_{x p(s)}(s)\right)$, $\left(m_{x p(t)}(t), t\right) \in R \subset \rho_{\nu_{0} \phi}$, and, since $\left(m_{x p(s)}(s), m_{x p(t)}(t)\right) \in \rho_{\nu_{0} \phi},(s, t) \in \rho_{\nu_{0} \phi^{*}}$. Thus, $\rho_{\eta} \subset \rho_{\nu \infty \phi}$.

Suppose now that $(s, t) \in \rho_{\nu_{0} \phi}$. Then $\phi(s) \in \phi(t) S \cap S \phi(t)$, and so there is $b \in S^{\prime}$ with $\phi(s)=\phi(t b)$. Hence, $(s, t b) \in R$, and so $\eta(s)=\eta(t b)=\eta(t) \eta(b)$. Therefore, $\eta(s) \leqq_{R} \eta(t)$, and similar arguments yield $\eta(s) \leqq_{\mathcal{L}} \eta(t), \eta(t) \leqq_{R} \eta(s)$, and $\eta(t) \leqq_{\mathcal{Q}}$ 
$\eta(s)$. Thus, $(\eta(s), \eta(t)) \in \mathbb{R} \cap \mathcal{L}=\mathcal{H}_{T}$, and, since all the subgroups of $T$ are trivial, $\eta(s)=\eta(t)$, whence $(s, t) \in \rho_{\eta}$, and $\rho_{\nu \circ \phi} \subset \rho_{\eta}$. We therefore have the desired result.

The author is indebted to Professor Thomas Hays for the argument for the continuity of the function $f$ in the proof of the necessity in this theorem.

We note that, in view of Lemmas 1.3 and 1.4, we have really characterized those compact semigroups $S$ with $\mathcal{H}$ a congruence on $S$ and $S / \mathcal{H}$ a tree with idempotent endpoints in which the idempotents commute as semigroups over trees.

\section{REFERENCES}

1. K. W. Folley, Semigroups, Proc. Sympos., Semigroups, Wayne State University, Detroit, Mich. (June 27-29, 1968), Academic Press, New York, 1969. MR 41 \#3637.

2. K. H. Hofmann and M. W. Mislove, The centralizing theorem for left normal groups of units in compact monoiods, Semigroup Forum 3(1971), 31-42.

3. K. H. Hofmann and P. S. Mostert, Elements of compact semigroups, Merrill, Columbus, Ohio, 1966. MR 35 \#285.

4. R. P. Hunter, On the semigroup struet ure of continua, Trans. Amer. Math. Soc. 93 (1959), 356-368. MR 22 \#82. 1136.

5. J. L. Kelley, General topology, Van Nostrand, Princeton, N. J., 1955. MR 16, $\# 1499$.

6. R. J. Koch, Threads in compact semigroups, Math. Z. 86 (1964), 321-316. MR 30

7. R. J. Koch and I. S. Krule, Weak eutpoint ordering on hereditarily unicoherent continua, Proc. Amer. Math. Soc. 11 (1960), 679-681. MR 22 \#11356.

8. R. J. Koch and A. D. Wallace, Maximal ideals in compaet semigroups, Duke Math. J. 21 (1954), 681-685. MR 16, 112.

9. M. W. Mislove, Four problems about compaet semigroups, Doctoral Dissertation, University of Tennessee, 1969.

10. L. Nachbin, Sur les espaces topologiques ordonnés, C. R. Acad. Sci. Paris 226 (1948), 381-382; Sur les espaces uniformisables ordonnés, C. R. Acad. Sci. Paris 226 (1948), 547; Sur les espaces ordonnés, C. R. Acad. Sci. Paris 226 (1948), 774-775; English transl. in Topology and order, Van Nostrand Math. Studies, Van Nostrand, Princeton, N. J., 1965. MR 9, 367; 9, 455; 36 \#2125.

11. R. C. Phillips, Interval elans with non-degenerate kernel, Proc. Amer. Math. Soc. 14 (1963), 396-400. MR 26 \#5547.

12. L. E. Ward, Jr., On dendritic sets, Duke Math. J. 25 (1958), 505-513. MR 20 \#4818.

13. -, Mobs, trees, and fixed points, Proc. Amer. Math. Soc. 8 (1957), 798-804. MR $20 \# 3516$.

14. - A note on dendrites and trees, Proc. Amer. Math. Soc. 5 (1954), 992-994. MR $17,180$.

15. M. *. Mislove, The exist ence of $\operatorname{Irr}(X)$, Trans. Amer. Math. Soc. 175 (1973), 123-140.

DEPARTMENT OF MATHEMATICS, TULANE UNIVERSITY, NEW ORL EANS LOUISIANA 70118 